\title{
Avaliação da função diastólica por meio de Doppler tecidual pulsado e colorido em gatos da raça Maine Coon geneticamente testados para a mutação no gene MyBPC-A31P ${ }^{1}$
}

\author{
Arine Pellegrino ${ }^{2 *}$, Alexandre G.T. Daniel ${ }^{3}$, Guilherme G. Pereira ${ }^{4}$, Francisco F. Lima \\ Júnior², Paula H. Itikawa² e Maria Helena M.A. Larsson²
}

\begin{abstract}
Pellegrino A., Daniel A.G.T., Pereira G.G., Júnior F.F.L., Itikawa P.H. \& Larsson M.H.M.A. 2014. [Assessment of diastolic function by pulsed and color tissue Doppler echocardiography in Maine Coon cats tested for MyBPC-A31P mutation.] Avaliação da função diastólica por meio de Doppler tecidual pulsado e colorido em gatos da raça Maine Coon geneticamente testados para a mutação no gene MyBPC-A31P. Pesquisa Veterinária Brasileira 34(3):290-300. Departamento de Clínica Médica, Faculdade de Medicina Veterinária e Zootecnia, Universidade de São Paulo, Av. Prof. Dr. Orlando Marques de Paiva 87, São Paulo, SP, 05508-270, Brazil. E-mail: arinepel@yahoo.com.br

Hypertrophic cardiomyopathy ( $\mathrm{HCM}$ ) is the most common feline heart disease and is characterized by increased cardiac mass with a hypertrophied nondilated left ventricle. Myocardial dysfunction occurs in cats with HCM but less is known about dysfunctions in initial stages of HCM. A mutation in MYBPC-A31P gene has been identified in a colony of Maine Coon cats with HCM. However, the close correlation between genotype and phenotype still be inconclusive. Myocardial analysis by tissue Doppler imaging (TDI) is a noninvasive echocardiographic method to assess systolic and diastolic function that is more sensitive than conventional echocardiography. To evaluate diastolic and systolic function in cats with mutation, with or without ventricular hypertrophy, Maine Coon cats ( $n=57)$ were screened for mutation and examined with both echocardiography and TDI (pulsed tissue Doppler and color tissue Doppler methods). Then, were phenotypically classified in: normal $(n=45)$, suspects $(n=7)$ and HCM group $(n=5)$; and genotypically classified in: negative $(n=28)$, heterozygous $(n=26)$ and homozygous group $(n=3)$. Myocardial velocities (by pulsed and color tissue Doppler imaging) measured in the basal and mildventricular segment of the interventricular septal wall (IVS), left ventricular free wall (LVW), left ventricular anterior wall (LVAW), left ventricular posterior wall (LVPW) and radial segment of LVW, was compared among different groups. A decreased longitudinal Em velocities (pulsed tissue Doppler) at the mildventricular segment of LVW was observed in HCM cats compared with suspects and normal cats. A decreased longitudinal Em/Am (color tissue Doppler) at the basal segment of IVS was observed in HCM cats compared with suspects and normal cats. A significant increased longitudinal E/Em (color tissue Doppler) at the basal segment of IVS was observed in HCM cats compared with suspects and normal cats. And a significant decreased longitudinal Sm (color tissue Doppler) at the basal segment of the LVW was observed in heterozygous cats compared with negative cats, both without hypertrophy. There was a positive correlation between summated early and late diastolic velocities (Em/Am) and heart rate; and a positive correlation between $\mathrm{Sm}$ and Em velocities and heart rate,
\end{abstract}

\footnotetext{
${ }^{1}$ Recebido em 23 de outubro de 2013.

Aceito para publicação em 18 de março de 2014.

${ }^{2}$ Departamento de Clínica Médica, Faculdade de Medicina Veterinária e Zootecnia, Universidade de São Paulo, Av. Prof. Dr. Orlando Marques de Paiva 87, Cidade Universitária, São Paulo, SP 05508-270, Brasil. *Autor para correspondência: arinepel@yahoo.com.br
}

\footnotetext{
${ }^{3}$ Departamento de Clínica Médica, Universidade Metodista de São Paulo, Av. Dom Jaime de Barros Câmara 100, Planalto, São Bernardo do Campo, SP 09895-400, Brasil.

${ }^{4}$ Departamento de Pós-Graduação, Universidade Santo Amaro (Unisa), Av. Prof. Enéas de Siqueira Neto 340, Jardim das Imbuias, São Paulo, SP 04829-300.
} 
both in pulsed and in color TDI. TDI analyses are a new, valuable and reproducible method in cats that alone is not able to identify cats with mutation before myocardial hypertrophy. Despite high expectations regarding the use of TDI for early identification of individuals with HCM, there is still need for larger studies with greater numbers of individuals.

INDEX TERMS: Hypertrophic cardiomyopathy, cats, Maine Coon, echocardiography, pulsed tissue Doppler, color tissue Doppler.

RESUMO.- A cardiomiopatia hipertrófica (CMH) é a principal cardiopatia dos felinos e é caracterizada por hipertrofia miocárdica concêntrica, sem dilatação ventricular. Disfunções miocárdicas ocorrem em gatos com $\mathrm{CMH}$, mas pouco se conhece a respeito destas alterações nos estágios iniciais da afecção. Em gatos da raça Maine Coon, a mutação no gene MyBPC-A31P está relacionada com a CMH de origem familial, porém, a correlação exata entre o genótipo e o fenótipo ainda é inconclusiva. A ecocardiografia tecidual é uma modalidade não invasiva que permite avaliação da função miocárdica e é mais sensível que a ecocardiografia convencional. Para avaliar as funções sistólica e diastólica, antes ou após a ocorrência de hipertrofia ventricular, gatos da raça Maine Coon $(n=57)$, geneticamente testados para a mutação, foram avaliados por meio de ecocardiografias convencional e tecidual (nas modalidades Doppler tecidual pulsado e Doppler tecidual colorido). Posteriormente, foram fenotipicamente classificados em: normais $(n=45)$, suspeitos $(\mathrm{n}=7)$ e acometidos pela $\mathrm{CMH}(\mathrm{n}=5)$; e genotipicamente classificados em: negativos $(n=28)$, heterozigotos $(n=26)$ e homozigotos para a mutação $(n=3)$. Valores de velocidades miocárdicas (Doppler tecidual pulsado e colorido) medidos na região basal e média do septo interventricular (SIV), da parede livre do ventrículo esquerdo (PVE), da parede anterior do ventrículo esquerdo (PAVE), da parede posterior do ventrículo esquerdo (PPVE) e do segmento radial da PVE, foram comparados nos diferentes grupos. Observou-se que as velocidades longitudinais Em (Doppler tecidual pulsado) na região média da PVE foram menores nos gatos com CMH quando comparados com suspeitos e normais. Os valores de Em/Am (Doppler tecidual colorido), na região basal do SIV, foram inferiores nos gatos com CMH quando comparados com suspeitos e normais. A relação E/Em (Doppler tecidual colorido), na região basal do SIV, foi maior nos gatos com CMH em relação aos suspeitos e normais, enquanto que os valores de Sm (Doppler tecidual colorido), em região basal da PVE, foram menores nos gatos heterozigotos quando comparados com os negativos, ambos sem hipertrofia ventricular. Observou-se correlação positiva entre a ocorrência de fusão das ondas Em e Am e a frequência cardíaca, assim como correlação positiva entre valores de $\mathrm{Sm}$ e Em e a frequência cardíaca (Doppler tecidual pulsado e colorido). A ecocardiografia tecidual é uma nova modalidade ecocardiográfica reprodutível em gatos que, isoladamente, não permite diferenciar gatos portadores da mutação antes do desenvolvimento de hipertrofia ventricular. Apresenta utilidade como auxílio no diagnóstico em fases iniciais, mas, apesar da expectativa para a identificação precoce de indivíduos portadores da $\mathrm{CMH}$, ainda há necessidade de estudos mais extensos e com maior número de indivíduos.
TERMOS DE INDEXAÇÃO: Cardiomiopatia hipertrófica, gatos, Maine Coon, ecocardiografia, Doppler tecidual pulsado, Doppler tecidual colorido.

\section{INTRODUÇÃO}

A CMH é caracterizada por hipertrofia ventricular esquerda, sem dilatação (Fox 1999, Chetboul et al. 2006a, McDonald et al. 2007). É a principal cardiopatia dos felinos, associando-se ao desenvolvimento de insuficiência cardíaca, tromboembolismo e morte súbita (Sampedrano et al. 2009). É uma afecção geneticamente e fenotipicamente heterogênea e, em felinos Maine Coon, pode estar associada à mutação autossômica dominante no gene MYBPC-A31P (Connolly et al. 2003, Haggström 2003, Baty 2004).

0 espessamento miocárdico determina o aumento na rigidez ventricular e, consequentemente, o desenvolvimento de alterações no relaxamento (McDonald 2005, McDonald Kittleson \& Kass 2008). Pressões diastólicas aumentadas são necessárias devido à rigidez e à menor distensibilidade ventricular (Severino et al. 2000, Chetboul et al. 2006b, Gundler Tidholm \& Haggstrõm 2008). 0 ventrículo esquerdo, mais rígido, requer pressões de enchimento maiores e, consequentemente, as pressões diastólicas atriais e ventriculares aumentam (Kato et al. 2007, Ware 2007, Koffas et al. 2008).

A ecocardiografia convencional é o exame mais utilizado para avaliação da anatomia e função cardíacas (Thomas et al. 1992 Wynne \& Braunwald 2003) e é o melhor meio diagnóstico não invasivo para a diferenciação de $\mathrm{CMH}$ de outras cardiomiopatias (Chetboul et al. 2006a, Chetboul et al. 2006b, Simpson et al. 2007, Ferasin 2009a). A identificação dos quatro componentes básicos (hipertrofia, disfunção diastólica, regurgitação mitral e obstrução de via de saída) e a correta interpretação das informações permite abordagem terapêutica adequada (Graziano \& Acquatella 2007, Ferasin 2009a).

O Doppler tecidual é uma ferramenta diagnóstica que permite avaliação não invasiva do coração, possibilitando a quantificação da função miocárdica regional ou global e a avaliação do movimento miocárdico (Koffas et al. 2008). É sensível e específico para avaliação de disfunções miocárdicas, sem apresentar influências de pré ou pós-carga (Oki et al. 2000, McDonald et al. 2007). Pode auxiliar na detecção de anormalidades em indivíduos geneticamente afetados para a CMH, antes do desenvolvimento de alterações à ecocardiografia convencional (Abecasis et al. 2009, Toro et al. 2009).

A utilização do Doppler tecidual em humanos com CMH permite avaliação de disfunção diastólica antes mesmo do desenvolvimento de hipertrofia ventricular (Toro et al. 2009). É uma ferramenta promissora que pode auxiliar na 
detecção precoce de anormalidades em indivíduos geneticamente afetados para a CMH (Abecasis et al. 2009).

Gatos geneticamente predispostos à $\mathrm{CMH}$ geralmente desenvolvem a enfermidade apresentando manifestações de insuficiência cardíaca congestiva ou morte súbita entre 1,5 a 3 anos de idade. 0 diagnóstico é basicamente realizado pela ecocardiografia convencional, com a detecção de hipertrofia miocárdica segmentar ou difusa. A detecção da CMH precoce, antes do desenvolvimento de hipertrofia, é de grande interesse, já que a identificação de gatos afetados permite sua exclusão dos programas de cruzamentos (reduzindo a ocorrência da CMH de origem familial). Além disso, o diagnóstico precoce pode auxiliar no manejo clínico da cardiomiopatia hipertrófica (Chetboul et al. 2006b).

Alterações no Doppler tecidual em gatos jovens, antes do desenvolvimento de alterações detectáveis na ecocardiografia convencional, podem auxiliar no diagnóstico precoce da $\mathrm{CMH}$, justificando a importância do conhecimento desta nova modalidade ecocardiográfica e a realização do presente trabalho científico.

\section{MATERIAL E MÉTODOS}

Foram incluídos neste estudo prospectivo e cego, 57 gatos Maine Coon, machos e fêmeas, após aprovação da Comissão de Bioética (Protocolo 1658/2009). Todos os animais foram avaliados por anamnese, exame físico e exames laboratoriais (hemograma, uréia, creatinina, enzimas hepáticas, proteína total, albumina, sódio, potássio, cálcio total, fósforo e hormônio tireoidiano (T4 total)). Foram realizados exames eletrocardiográfico, radiográfico e mensuração da pressão arterial de acordo com metodologias, respectivamente, estabelecidas por Tilley (1992), Buchanan \& Bücheler (1995) e Brown et al. (2007).

De acordo com critérios de exclusão, não participaram do estudo animais com hipertensão arterial sistêmica, nefropatia e/ou hipertireoidismo; e gatos que apresentaram qualquer outra enfermidade que pudesse inferir no sistema cardiovascular, totalizando sete animais excluídos.

Amostras de sangue total foram enviadas ao laboratório Veterinary Cardiac Genetics Laboratory da Washington State University para a identificação da mutação no gene MyBPC-A31P por meio de genotipagem, pela técnica de PCR (Meurs et al. 2007). De acordo com os resultados obtidos, os animais foram classificados em:

$$
\begin{aligned}
& \text { - negativos }(n=28) ; \\
& \text { - heterozigotos }(n=26) ; \\
& \text { - homozigotos }(n=3) .
\end{aligned}
$$

A informação referente à definição genética dos gatos só foi conhecida após o término do estudo, com a finalidade de reduzir interferências subjetivas nas interpretações dos exames (blinded study).

0 exame ecocardiográfico foi realizado utilizando-se ecocardiógrafo modelo Vivid 7 Expert (General Eletric Co.- GE), provido de software para realização de ecocardiografia tecidual, conforme recomendações da Echocardiography Committee of the Specialty of Cardiology-American College of Veterinary Internal Medicine e American Society of Echocardiography (Thomas et al. 1992, Boon 2011). Os animais, livres de sedação e/ou tranquilização, foram mantidos em monitoração eletrocardiográfica simultânea durante o ecocardiograma.

Foram realizadas, no mínimo, três determinações de cada parâmetro avaliado nas diferentes fases do ciclo cardíaco, considerando-se a média dos valores obtidos. As imagens para a mensuração do ventrículo esquerdo foram adquiridas na janela paraesternal direita, corte transversal, na altura da inserção das cordoalhas tendíneas nos músculos papilares (modo M). A presença de hipertrofia miocárdica foi definida quando a espessura diastólica do SIV (SIVd) e/ou da parede livre do ventrículo esquerdo (PVEd) era igual ou superior a 0,6 cm (Sampedrano et al. 2006, Sampedrano et al. 2009). Os animais com espessuras diastólicas menores que $0,5 \mathrm{~cm}$ foram considerados normais; e os gatos com valores de espessuras diastólicas entre 0,5 e 0,6 foram considerados suspeitos para a CMH. A hipertrofia concêntrica foi considerada simétrica quando a relação SIVd/PVEd estava entre 0,7 e 1,3. $\mathrm{Na}$ presença de hipertrofia assimétrica, fez-se a mensuração da hipertrofia segmentar pelo modo bidimensional. As mensurações do diâmetro da raiz da aorta (Ao) e do diâmetro do átrio esquerdo (AE) foram realizadas pelo modo bidimensional (Abbott \& Mclean 2006), janela paraesternal direita, corte transversal, em região de base cardíaca. Considerou-se aumento do $\mathrm{AE}$ quando a relação AE/Ao foi superior a 1,5 (Wess Sakar \& Hartmann 2010).

Os parâmetros avaliados pelo Doppler pulsado foram velocidade máxima e gradiente de pressão do fluxo da aorta e do fluxo da artéria pulmonar; velocidade máxima da onda E e da onda A do fluxo transmitral, relação E/A, tempo de desaceleração da onda E e tempo de relaxamento isovolumétrico (TRIV).

Após a realização do exame ecocardiográfico, os animais foram classificados, quanto à $\mathrm{CMH}$, em:

$$
\begin{aligned}
& \text { - normais }(n=45) ; \\
& \text { - suspeitos }(n=7) ; \\
& \text { - animais com CMH }(n=5) \text {. }
\end{aligned}
$$

Além disso, com a finalidade de avaliar os indivíduos antes do desenvolvimento de hipertrofia ventricular, os gatos normais foram classificados quanto ao genótipo em:

$$
\begin{aligned}
& \text { - normais e negativos }(n=25) \text {; } \\
& \text { - normais e heterozigotos }(n=19) \text {; } \\
& \text { - normais e homozigotos }(n-1) \text {. }
\end{aligned}
$$

Na modalidade Doppler tecidual pulsado, a avaliação da função miocárdica longitudinal foi realizada na imagem apical quatro câmaras e apical duas câmaras; e a avaliação da função radial foi realizada no corte transverso à altura dos músculos papilares. Os segmentos avaliados foram: região basal e média do SIV e da PVE no corte apical quatro câmaras (longitudinal), região basal e média da parede anterior (PAVE) e posterior do ventrículo esquerdo (PPVE) no corte apical duas câmaras (longitudinal) e na região do endocárdio e epicárdio à altura dos músculos papilares na parede lateral (transverso). Durante a avaliação, realizou-se monitorização eletrocardiográfica simultânea e padronizou-se o tamanho da amostra do Doppler tecidual pulsado em 1 a $2 \mathrm{~mm}$ (Koffas et al. 2006, McDonald et al. 2007). Em cada segmento analisado, foram obtidas as frequências cardíacas instantâneas, as velocidades miocárdicas máximas das ondas de enchimento ventricular rápido (Em), contração atrial ou enchimento ventricular lento (Am) e sistólica (Sm) (Fig.1). Além disso, a relação Em/Am e a relação E/ Em também foram determinadas, de acordo com Chetboul et al. (2006a).

Na modalidade Doppler tecidual colorido, a avaliação da função miocárdica longitudinal também foi realizada na imagem apical quatro câmaras e apical duas câmaras; e a avaliação da função radial, no corte transverso à altura dos músculos papilares. Os segmentos avaliados foram os mesmos realizados para o Doppler tecidual pulsado. Durante a avaliação (realizada de modo "off-line"), realizou-se monitorização eletrocardiográfica simultânea, utilizou-se a ferramenta "marcador de eventos" para identificar o momento de abertura e fechamento da valva aórtica, e fez-se a mensuração da frequência cardíaca instantânea (para observar a 
influência da mesma em cada medida avaliada). Padronizou-se o tamanho da amostra do Doppler tecidual colorido em $2 \times 2 \mathrm{~mm}$ (Sampedrano et al. 2009). Em cada segmento analisado, foram obtidas as frequências cardíacas instantâneas, as velocidades miocárdicas máximas das ondas de enchimento ventricular rápido (Em), contração atrial ou enchimento ventricular lento (Am) e sistólica (Sm) (Fig.2). Além disso, a relação Em/Am, a relação E/Em e o gradiente de velocidade miocárdica longitudinal (diferença de velocidades entre a base e o meio da parede e do septo interventricular) também foram determinados (Fig.3), de acordo com Chetboul et al. (2006a). Todos os parâmetros foram avaliados pelo mesmo ecocardiografista; e fez-se a análise de variação intraobservador.

À análise estatística, utilizou-se o Shapiro-Wilks para o teste de normalidade. Média, desvio padrão, valor mínimo, valor máximo e mediana foram calculados nas variáveis com distribuição normal. Para comparar as médias das variáveis contínuas utilizou-se o p-valor do teste T-student e/ou Mann-Whitney (dois grupos); e

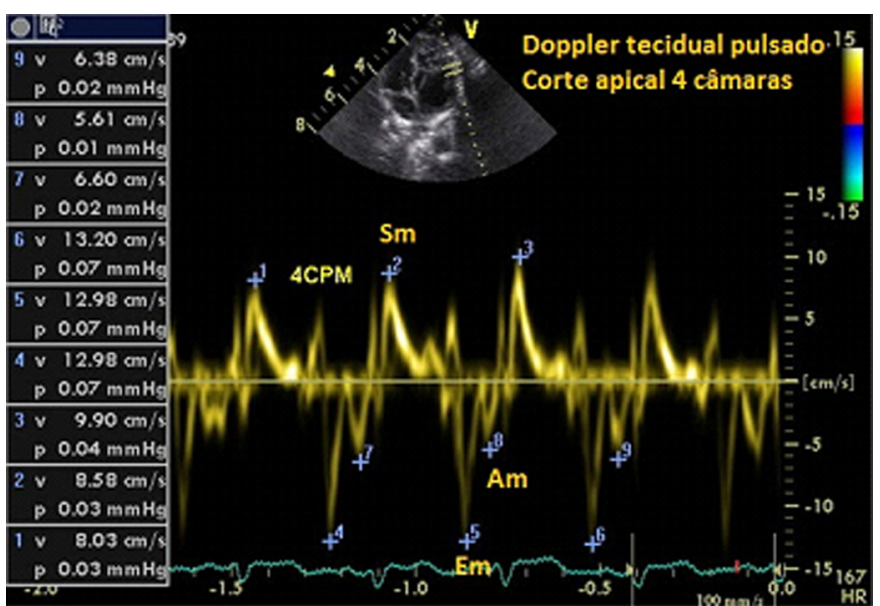

Fig.1. Doppler tecidual pulsado em região média de parede livre de ventrículo esquerdo em corte apical quatro câmaras. Am = onda $\mathrm{Am} ; \mathrm{Em}$ = onda $\mathrm{Em} ; \mathrm{Sm}$ = onda $\mathrm{Sm} ; 4 \mathrm{CPM}$ = região média de parede livre de ventrículo esquerdo em corte apical quatro câmaras.

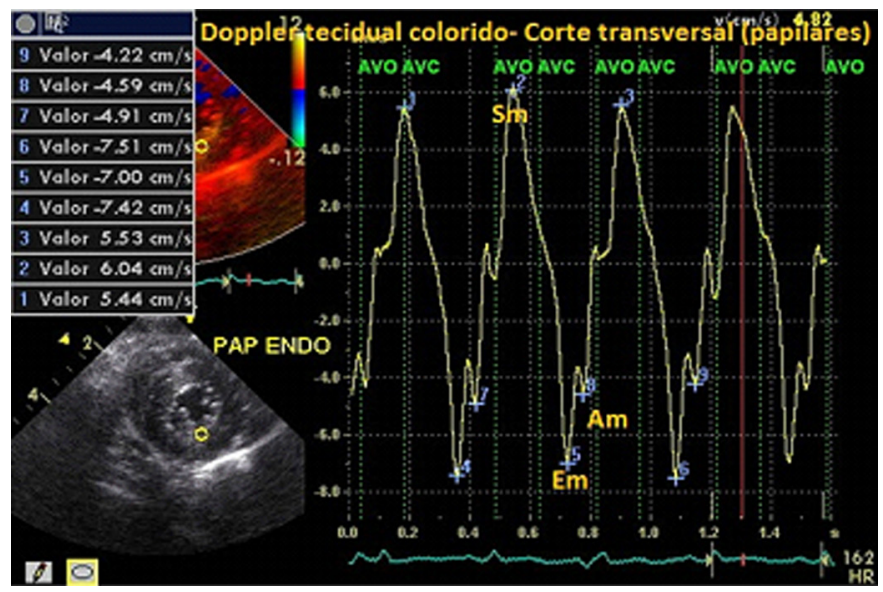

Fig.2. Doppler tecidual colorido em região endocárdica de parede livre de ventrículo esquerdo em corte transversal na altura dos músculos papilares. $\mathrm{Am}=$ onda $\mathrm{Am} ; \mathrm{Em}=$ onda $\mathrm{Em} ; \mathrm{Sm}=$ onda Sm; PAP ENDO = região endocárdica de parede livre de ventrículo esquerdo em corte transversal na altura dos músculos papilares.

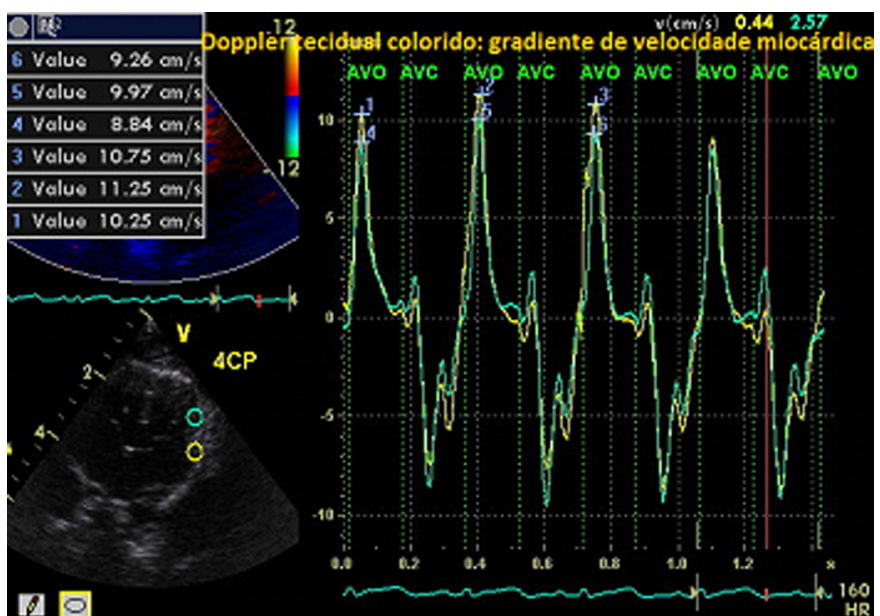

Fig.3. Gradiente de velocidade miocárdica- Doppler tecidual colorido em região de parede livre de ventrículo esquerdo em corte apical quatro câmaras. AVC = fechamento da valva aórtica; AVO = abertura da valva aórtica; $4 \mathrm{CP}=$ região de parede livre de ventrículo esquerdo em corte apical quatro câmaras.

utilizou-se o p-valor da ANOVA e/ou Kruskal-Wallis (três ou mais grupos). Devido ao baixo número de homozigotos, os mesmos foram desconsiderados nas avaliações estatísticas. Utilizaram-se os testes de Mann-Whitney e Kruskal-Wallis no lugar dos respectivos testes T e ANOVA quando os testes de normalidade foram rejeitados em, pelo menos, um grupo ou quando existiram poucos dados (Siegel \& Castellan 1988, Neter et al. 1996). Para as variáveis categóricas, utilizaram-se tabelas de frequência e o teste Qui-Quadrado de Pearson e/ou o teste exato de Fisher. Para a avaliação das correlações, utilizou-se o coeficiente de correlação de Pearson. Para a avaliação da variabilidade intraobservador, utilizou-se o teste $\mathrm{T}$ pareado para diferença igual a zero. 0 nível de significância aplicado nos testes foi de 5\%, sempre considerando hipóteses alternativas bicaudais.

\section{RESULTADOS}

Dos 57 gatos, 23 eram machos (40,35\%) e 34 eram fêmeas (59,65\%). 0 peso corpóreo variou de 3,1 a 7,9 $\mathrm{kg}(5,11 \pm 1,26$ $\mathrm{kg})$, com média de 6,20 $0,81 \mathrm{~kg}(4,9$ a 7,9 kg) nos machos e 4,37 $\pm 0,93 \mathrm{~kg}(3,1$ a $6,7 \mathrm{~kg})$ nas fêmeas. A média do peso dos machos foi maior que das fêmeas $(\mathrm{P}<0,0001)$. Não foram observadas diferenças, quanto ao peso, entre animais inteiros e castrados. A faixa etária variou de oito a 89 meses $(33,72 \pm 17,22$ meses $)$ e não houve diferença entre a média de idade de machos e fêmeas.

Quanto à classificação genotípica, 28 gatos eram negativos para a mutação $(49,13 \%), 26$ heterozigotos $(45,61 \%)$ e apenas 3 homozigotos (5,26\%). Os homozigotos foram excluídos da análise estatística; e não foram observadas diferenças em relação ao peso, ao sexo e à faixa etária dos animais.

Quanto à classificação fenotípica, 45 gatos foram considerados normais (78,95\%), sete suspeitos $(12,28 \%)$ e cinco acometidos pela $\mathrm{CMH}(8,77 \%)$ (Fig.4). Não foram observadas diferenças em relação ao sexo e à faixa etária dos animais. Porém, os normais apresentaram peso corpóreo inferior aos suspeitos $(\mathrm{P}<0,001)$ e acometidos $(\mathrm{P}<0,05)$.

A distribuição dos gatos, quanto à classificação genotípica e fenotípica, está descrita no Quadro 1. 


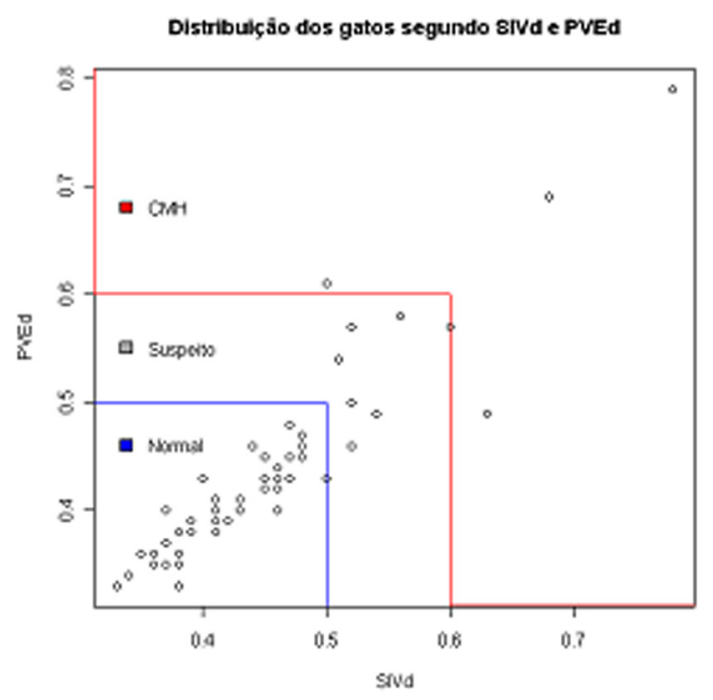

Fig.4. Distribuição dos animais, segundo espessuras diastólicas de septo interventricular (SIVd; em $\mathrm{cm}$ ) e de parede livre de ventrículo esquerdo (PVEd; em $\mathrm{cm}$ ), segundo classificação fenotípica (normais, suspeitos e com CMH)- São Paulo, 2010.

Quadro 1. Distribuição de gatos da raça Maine Coon, segundo genótipo e fenótipo. São Paulo, 2010

\begin{tabular}{lccc}
\hline Classificação & $\begin{array}{c}\text { Negativos } \\
(\mathrm{n}=28)\end{array}$ & $\begin{array}{c}\text { Heterozigotos } \\
(\mathrm{n}=26)\end{array}$ & $\begin{array}{c}\text { Homozigotos } \\
(\mathrm{n}=3)\end{array}$ \\
\hline Normais $(\mathrm{n}=45)$ & $\mathrm{n}=25$ & $\mathrm{n}=19$ & $\mathrm{n}=1$ \\
Suspeitos (n=7) & $\mathrm{n}=3$ & $\mathrm{n}=3$ & $\mathrm{n}=1$ \\
CMH (n=5) & $\mathrm{n}=0$ & $\mathrm{n}=4$ & $\mathrm{n}=1$ \\
Total (n=57) & $\mathrm{n}=28$ & $\mathrm{n}=26$ & $\mathrm{n}=3$
\end{tabular}

$\overline{\mathrm{CMH}}=$ cardiomiopatia hipertrófica; $\mathrm{n}=$ número de animais .

Para investigar alterações precoces (antes da hipertrofia ventricular), fez-se a comparação dentro do grupo dos gatos fenotipicamente normais em relação ao genótipo. A análise estatística foi realizada entre indivíduos negativos normais e heterozigotos normais. Não foram observadas diferenças quanto ao peso, idade e distribuição sexual nestes grupos.

Na avaliação do fluxo transmitral, houve fusão de ondas E e A, impedindo avaliação, em 26 animais (45,61\%). Observou-se correlação positiva entre a ocorrência da fusão e a frequência cardíaca $(\mathrm{P} \leq 0,008)$.

Todos os acometidos pela CMH apresentaram a forma simétrica da afecção, com relação SIVd/PVEd variando entre 0,82 e 1,29. De acordo com Wess Sakar \& Hartmann (2010), onde valores de SIVd e/ou de PVEd inferiores a 0,65 cm são considerados hipertrofia discreta, valores entre $0,65 \mathrm{~cm} \mathrm{e}$ $0,70 \mathrm{~cm}$, hipertrofia moderada; e valores superiores a 0,70 $\mathrm{cm}$, hipertrofia importante, a CMH foi considerada de grau discreto em três heterozigotos; de grau moderado num homozigoto; e de grau importante num heterozigoto.

Quanto à classificação genotípica, valores de velocidade máxima (e gradiente de pressão) do fluxo aórtico foram maiores nos heterozigotos em relação aos negativos $(\mathrm{P}<0,05)$. Nos demais parâmetros ecocardiográficos, não foram observados alterações significativas.
Quanto à classificação fenotípica, o diâmetro do AE foi maior nos acometidos pela CMH em relação aos normais $(\mathrm{P}<0,05)$. Observaram-se diferenças estatísticas no TRIV, que foi maior nos acometidos em relação aos suspeitos $(\mathrm{P}<0,05)$ e aos normais $(\mathrm{P}<0,001)$; e na velocidade do fluxo aórtico, que foi maior nos acometidos em relação aos suspeitos $(\mathrm{P}<0,001)$ e aos normais $(\mathrm{P}<0,001)$. Nos demais parâmetros, não foram observadas alterações.

Ao avaliar o grupo dos fenotipicamente normais, não foram observadas diferenças estatísticas nos diferentes parâmetros de ecocardiografia convencional.

Quanto à avaliação genotípica, não foram observadas diferenças estatísticas entre os parâmetros de Doppler tecidual pulsado e Doppler tecidual colorido entre gatos negativos e gatos heterozigotos para a mutação no gene MyBPC-A31P.

Quanto à avaliação fenotípica, utilizando-se o Doppler tecidual pulsado, observaram-se diferenças significativas entre os valores de Em apenas no corte apical quatro câmaras, em região média da parede livre do ventrículo esquerdo (4CPM). Neste corte, os valores de Em foram menores nos gatos acometidos pela $\mathrm{CMH}$ em relação aos suspeitos, e destes em relação aos fenotipicamente normais $(\mathrm{P}=0,001)$. Também foram observadas diferenças estatísticas em relação à frequência cardíaca na região de base da parede anterior do ventrículo esquerdo, em corte apical duas câmaras (2CAB), com os valores dos acometidos pela CMH menores que os dos suspeitos, e inferiores aos animais fenotipicamente normais $(\mathrm{P}=0,03)$.

Ao avaliar apenas o grupo dos animais fenotipicamente normais (45 gatos: 25 negativos, 19 heterozigotos e um homozigoto para a mutação), não foram observadas diferenças estatísticas nos diferentes parâmetros de ecocardiografia na modalidade Doppler tecidual pulsado.

As velocidades miocárdicas sistólicas e diastólicas avaliadas pelo Doppler tecidual pulsado foram maiores nas regiões de base em relação às regiões médias do miocárdio, e maiores no endocárdio em relação ao epicárdio, em todos os grupos estudados, exceto no corte apical duas câmaras, em região de parede posterior, na avaliação quanto ao fenótipo.

A ocorrência de fusão das ondas diastólicas Em e Am, em qualquer corte ecocardiográfico avaliado, esteve significativamente correlacionada com a frequência cardíaca $(\mathrm{P} \leq 0,008)$. Quanto maior a frequência cardíaca do animal avaliado, maior a ocorrência de fusão das ondas Em e Am. Também foi observada correlação positiva entre a frequência cardíaca e as velocidades miocárdicas $\mathrm{Sm}$ e Em em diversos segmentos avaliados. Quanto maior a frequência cardíaca, maiores as velocidades sistólica (Sm) e diastólica (Em) avaliadas pelo Doppler tecidual pulsado.

$\mathrm{Na}$ investigação de possível correlação negativa entre a espessura diastólica do septo interventricular e/ou da parede livre de ventrículo esquerdo e valores da relação Em/ Am (SIVd e/ou PVEd versus Em/Am), observou-se correlação negativa estatisticamente significativa $(\mathrm{P}=0,05$; correlação de Pearson=-0,37) apenas no corte apical quatro câmaras em região média da parede livre de ventrículo esquerdo (4CPM). 
Quanto à avaliação fenotípica, utilizando-se o Doppler tecidual colorido, observaram-se diferenças significativas entre os valores de $\mathrm{Em} / \mathrm{Am}$ apenas no corte apical quatro câmaras, em região de base do septo interventricular (4CSB). Neste corte, os valores de Em/Am foram menores nos gatos acometidos pela $\mathrm{CMH}$ em relação aos suspeitos e aos fenotipicamente normais $(\mathrm{P}=0,01)$.

Também foram observadas diferenças estatísticas na relação E/Em em região de base do septo interventricular, no corte apical quatro câmaras (4CSB), com os valores dos acometidos pela $\mathrm{CMH}$ maiores que os dos suspeitos e fenotipicamente normais $(\mathrm{P}=0,01)$.

Ao avaliar apenas o grupo dos animais fenotipicamente normais (45 gatos: 25 negativos, 19 heterozigotos e um homozigoto para a mutação), observaram-se diferenças estatísticas nas velocidades de Sm na região de base da parede livre do ventrículo esquerdo no corte apical quatro câmaras (4CPB) entre indivíduos negativos e heterozigotos. Os valores de $\mathrm{Sm}$ foram significativamente menores nos heterozigotos em relação aos negativos $(\mathrm{P}=0,02)$. Não houve diferenças estatísticas nos demais parâmetros ecocardiográficos avaliados.

As velocidades miocárdicas sistólicas e diastólicas avaliadas pelo Doppler tecidual colorido foram maiores nas regiões de base em relação às regiões médias do miocárdio, e maiores no endocárdio em relação ao epicárdio, em todos os grupos estudados.

A ocorrência de fusão das ondas diastólicas Em e Am, em qualquer corte ecocardiográfico avaliado, esteve significativamente correlacionada com a frequência cardíaca $(\mathrm{P}<0,001)$.

Também foi observada correlação positiva entre a frequência cardíaca e as velocidades miocárdicas Sm e Em em diversos segmentos avaliados. Quanto maior a frequência cardíaca, maiores as velocidades sistólica ( $\mathrm{Sm}$ ) e diastólica (Em) avaliadas pelo Doppler tecidual colorido. Porém, pode-se perceber que as ondas Em foram mais influenciadas pela frequência cardíaca que as ondas Sm neste método ecocardiográfico.

Na investigação de possível correlação negativa entre a espessura diastólica do septo interventricular e/ou da parede livre do ventrículo esquerdo e valores da relação Em/ Am (SIVd e/ou PVEd versus Em/Am), não foram observadas alterações estatisticamente significativas em nenhum segmento avaliado.

Não houve evidências de que as diferenças encontradas entre as medidas do Doppler tecidual pulsado e do Doppler tecidual colorido, realizadas em tempos diferentes mas pelo mesmo observador (variabilidade intraobservador), fossem distintas entre si (teste $\mathrm{T}$ pareado para diferença igual a zero). Portanto, a variabilidade intraobservador (tanto no método do Doppler tecidual pulsado quanto no Doppler tecidual colorido) foi pequena, reduzindo a possibilidade de vieses na interpretação dos parâmetros.

\section{DISCUSSÃO E CONCLUSÕES}

A população avaliada foi composta por Maine Coons geneticamente testados para a mutação no gene MyBPC-A31P. Dos 57 gatos, $50,87 \%$ eram positivos para a mutação
(45,61\% heterozigotos e 5,26\% homozigotos). De acordo com estudos de Meurs et al. $(2005,2007,2009)$ e Wess et al. (2010), a mutação no referido gene está presente em aproximadamente $34 \%$ dos Maine Coons de todo o mundo, porém poucos estudos correlacionam o genótipo com a ocorrência de $\mathrm{CMH}$ propriamente dita.

Dentre os animais avaliados, $8,77 \%$ já apresentava achados ecocardiográficos compatíveis com $\mathrm{CMH}$, todos com mutação no gene MyBPC-A31P (quatro heterozigotos e um homozigoto). Foram considerados suspeitos para a CMH, 12,28\% (7 animais) da população avaliada. Destes, quatro apresentavam a mutação e três eram negativos. A grande maioria dos animais apresentou-se normal à avaliação ecocardiográfica $(78,95 \%)$, apesar da existência de gatos heterozigotos e homozigotos neste grupo. Segundo os estudos de Meurs et al. (2005), McDonald et al. (2007) e Kittleson (2009), a CMH é uma afecção de herança autossômica dominante em gatos Maine Coon, porém a penetrância incompleta é comum neste tipo de mutação, tornando o diagnóstico ecocardiográfico mais difícil nos heterozigotos. Além disso, há mais de 400 mutações em 11 genes que codificam proteínas do sarcômero relacionadas à $\mathrm{CMH}$ e, portanto, a ausência da mutação no gene MyBPC-A31P não exclui a possibilidade da existência de outras mutações que possam causar cardiomiopatia (McTaggart 2002, Haggstrom 2003, Fries et al. 2008, Wess et al. 2010). Sabe-se que a CMH é uma doença geneticamente e fenotipicamente heterogênea (Kittleson et al. 1999, Haggstrom 2003, Batty 2004), o que pode dificultar a interpretação e a obtenção de conclusões no presente estudo.

A distribuição de machos e fêmeas foi homogênea nos grupos estudados. Porém, a prevalência de machos entre os suspeitos e acometidos foi significativamente maior $(60 \%$ dos gatos com CMH e 71,43\% dos suspeitos eram machos). Segundo Chetboul et al. (2006a) e Fuentes (2006), a CMH é mais relatada em machos de meia idade, mas também pode ocorrer em fêmeas, em jovens ou idosos. Alguns estudos não referem diferenças quanto ao sexo, mas há relatos de que as manifestações podem ser precoces e mais evidentes nos indivíduos masculinos (Ware 2007).

A média do peso dos machos foi maior que das fêmeas, o que pode ser justificado pelo padrão racial dos animais, onde machos são visivelmente maiores que fêmeas. Os animais normais apresentaram peso corpóreo inferior aos demais. Porém, havia mais fêmeas no grupo dos normais, justificando os valores encontrados.

Quanto à classificação genotípica, valores de velocidade do fluxo aórtico foram maiores nos heterozigotos em relação aos negativos. Porém, alguns destes pacientes também apresentavam alterações fenotípicas quanto à $\mathrm{CMH}$, o que pode interferir nos valores encontrados. Ao avaliar apenas o grupo dos normais, não foram observadas diferenças quanto ao genótipo. Assim, a ecocardiografia convencional não foi capaz de diferenciar indivíduos negativos ou portadores da mutação, antes do desenvolvimento de hipertrofia ventricular, fato já relatado, anteriormente, por outros autores (Chetboul et al. 2006a, Simpson et al. 2008, Ferasin 2009b, Sampedrano et al. 2009).

Já em relação à classificação fenotípica, o diâmetro do 
AE foi maior nos acometidos pela CMH em relação aos normais. Observaram-se diferenças no TRIV e na velocidade do fluxo aórtico, que foram maiores nos acometidos quando comparados aos suspeitos e normais. $\mathrm{Na} \mathrm{CMH}$, pode-se observar aumento no TRIV, devido à disfunção diastólica observada (alteração de relaxamento ventricular); e aumento na velocidade e no gradiente de pressão do fluxo aórtico, quando há obstrução dinâmica na via de saída do ventrículo esquerdo (Simpson et al. 2007, Ferasin 2009b). Segundo a literatura, o ecocardiograma é o melhor meio diagnóstico não invasivo para a diferenciação de CMH de outras cardiomiopatias e permite caracterização de anormalidades funcionais (Chetboul et al. 2006a, 2006b, Simpson et al. 2007, Gundler et al. 2008).

Na modalidade Doppler tecidual pulsado, não foram observadas diferenças estatísticas significativas entre indivíduos genotipicamente negativos e heterozigotos. Segundo o estudo de McDonald et al. (2007), que avaliou parâmetros de Doppler tecidual pulsado em gatos da raça Maine Coon normais ou portadores de mutação, com ou sem hipertrofia ventricular, os valores de velocidade de Em (região do anel mitral) sofreram redução de acordo com presença ou ausência de hipertrofia. Neste mesmo estudo, gatos genotipicamente afetados, mas sem hipertrofia, apresentaram valores intermediários de Em entre gatos normais e gatos genotipicamente afetados e com hipertrofia. Porém, o Doppler tecidual pulsado não foi capaz de diferenciar gatos geneticamente afetados antes do desenvolvimento de hipertrofia ventricular, à semelhança do que ocorreu no presente trabalho.

Ao avaliar apenas o grupo dos animais fenotipicamente normais, na tentativa de investigar possíveis alterações precoces antes do desenvolvimento de hipertrofia ventricular, não foram observadas diferenças estatísticas nos diferentes parâmetros do Doppler tecidual pulsado entre gatos negativos e heterozigotos para a mutação. Portanto, à semelhança dos resultados obtidos por McDonald et al. (2007), o Doppler tecidual pulsado não foi capaz de diferenciar gatos geneticamente afetados antes do desenvolvimento de hipertrofia ventricular.

Quanto à avaliação fenotípica, observaram-se diferenças significativas entre os valores de Em apenas no corte apical quatro câmaras, em região média da parede livre do ventrículo esquerdo (4CPM). Neste corte, os valores de Em (em Doppler tecidual pulsado) foram menores nos gatos acometidos pela $\mathrm{CMH}$ em relação aos suspeitos, e destes em relação aos fenotipicamente normais. Alguns estudos, com uso de ecocardiografia na modalidade Doppler tecidual pulsado, demonstraram que gatos com $\mathrm{CMH}$ apresentam diminuição das velocidades miocárdicas sistólicas e diastólicas, diminuição nos gradientes de velocidade, perda de uniformidade do movimento miocárdico (Koffas et al. 2006) e prolongamento do tempo de relaxamento isovolumétrico quando comparados com gatos normais (Koffas et al. 2008).

Segundo McDonald et al. (2006b, 2007) e Simpson et al. (2008), gatos com hipertrofia miocárdica causada pela CMH apresentaram menores velocidades de Em quando comparados com gatos normais, à semelhança do resultado obtido no trabalho atual.
Observou-se correlação positiva entre a frequência cardíaca e as velocidades miocárdicas $\mathrm{Sm}$ e Em em diversos segmentos avaliados. Quanto maior a frequência cardíaca, maiores foram as velocidades sistólica $(\mathrm{Sm})$ e diastólica (Em) no Doppler tecidual pulsado. Simpson et al. (2008), à semelhança dos resultados obtidos no presente trabalho, observaram correlação positiva entre a frequência cardíaca e a magnitude das velocidades de Em, Am e Sm no movimento radial e das velocidades de Am e Sm no movimento longitudinal miocárdio.

Na investigação de possível correlação negativa entre a espessura diastólica do septo interventricular e/ou da parede livre de ventrículo esquerdo e valores da relação Em/ Am (SIVd e/ou PVEdversus Em/Am), observou-se correlação negativa, estatisticamente significativa, apenas no corte apical quatro câmaras em região média da parede livre de ventrículo esquerdo (4CPM). Desta forma, pode-se perceber que quanto maior a espessura do septo e/ou da parede livre do ventrículo esquerdo, maior a possibilidade de detecção de disfunção diastólica ao Doppler tecidual pulsado. Segundo o trabalho de Koffas et al. (2006), a espessura da parede livre do ventrículo esquerdo também esteve negativamente correlacionada com a relação $\mathrm{Em} / \mathrm{Am}$ na região de base da parede livre em corte apical quatro câmaras.

$\mathrm{Na}$ interpretação do teste de variabilidade intraobservador, não houve evidências de que as diferenças encontradas entre as medidas do Doppler tecidual pulsado, realizadas em tempos diferentes e pelo mesmo observador, fossem distintas entre si. Portanto, a variabilidade intraobservador foi pequena, comprovando a boa repetibilidade do método que é descrita na literatura (Simpson et al. 2007, Yu et al. 2007).

Diferentemente de alguns trabalhos citados, como o de Koffas et al. (2006) que obteve um ponto de corte de velocidade miocárdica com sensibilidade e especificidade altas na identificação de gatos normais e gatos com $\mathrm{CMH}$, no presente trabalho não foi possível detectar um valor de velocidade miocárdica abaixo do qual a identificação da CMH e/ ou da mutação fosse alta. Portanto, mais estudos são necessários para estabelecer a utilidade do Doppler tecidual na detecção de anormalidades, antes do desenvolvimento de hipertrofia ventricular (Graziano \& Acquatella 2007).

Na modalidade Doppler tecidual colorido, quanto à avaliação genotípica, não foram observadas diferenças estatísticas entre gatos negativos e gatos heterozigotos para a mutação no gene MyBPC-A31P. Ao avaliar apenas o grupo dos animais fenotipicamente normais, na tentativa de investigar possíveis alterações precoces antes do desenvolvimento de hipertrofia ventricular, observaram-se diferenças estatísticas nas velocidades de Sm na região de base da parede livre do ventrículo esquerdo no corte apical quatro câmaras (4CPB) entre indivíduos negativos e heterozigotos. Os valores de $\mathrm{Sm}$ foram significativamente menores nos heterozigotos em relação aos indivíduos negativos. De acordo com Oki et al. (2000) e Toro et al. (2009), à semelhança do ocorrido no presente estudo, as velocidades miocárdicas sistólicas e diastólicas, medidas por Doppler tecidual, encontraram-se reduzidas em pessoas com mutação causadora de $\mathrm{CMH}$, sem hipertrofia ventricular. De 
acordo com Sampedrano et al. (2009), a velocidade miocárdica diastólica precoce (Em) no anel mitral, mensurada por Doppler tecidual, foi menor em Maine Coons homozigotos para a mutação em relação aos Maine Coons heterozigotos; e nestes em relação aos gatos normais. Porém, no presente trabalho, não se observaram diferenças estatísticas em relação às velocidades diastólicas precoces (Em).

Recentemente, um estudo mostrou que as velocidades miocárdicas sistólicas e diastólicas, medidas por Doppler tecidual colorido, encontram-se reduzidas em pessoas que têm mutação causadora de cardiomiopatia hipertrófica, mas que não têm hipertrofia ventricular, quando comparado a pacientes sadios (Nagueh et al. 2001, Gandjbakhchet al. 2010). Porém, de acordo com Gandjbakhch et al. (2010) e à semelhança do ocorrido no presente trabalho, velocidades do Doppler tecidual colorido isoladamente não permitiram a identificação dos pacientes carreadores da mutação antes do desenvolvimento da hipertrofia ventricular com boa sensibilidade e especificidade. Para permitir identificação destes portadores com maior acurácia, os autores desenvolveram um escore combinando parâmetros do Doppler tecidual colorido e alguns valores do ecocardiograma convencional. Outros estudos não encontraram diferenças em velocidades miocárdicas Em e Sm entre portadores sem hipertrofia e pacientes humanos normais. Segundo Pinto (2007), apesar da grande expectativa em relação ao uso do Doppler tecidual colorido para a identificação precoce de indivíduos portadores da $\mathrm{CMH}$, ainda há necessidade de estudos mais extensos e com maior número de indivíduos. No presente trabalho, observou-se valores menores de Sm (em 4CPB) nos heterozigotos em relação aos animais negativos, resultado interessante para uma futura investigação com maior número de animais na tentativa de identificação de animais portadores da mutação.

No presente estudo, não foram observadas alterações em velocidades diastólicas Em nem nas relações Em/Am entre indivíduos heterozigotos e negativos para a mutação. No estudo de Sampedrano et al. (2009), apesar da diminuição significativa na relação $\mathrm{Em} / \mathrm{Am}$ do segmento basal do septo interventricular em gatos Maine Coons heterozigotos para a mutação e sem hipertrofia, quando comparados com gatos normais sem hipertrofia, o genótipo heterozigoto não esteve sempre associado à hipertrofia miocárdica ou às alterações diastólicas importantes; e os gatos sem a mutação no gene MYBPC-A31P também desenvolveram hipertrofia, sugerindo outras causas genéticas envolvidas. Portanto, muitas das semelhanças entre as velocidades diastólicas encontradas no presente estudo devem-se, em parte, à grande variabilidade fenotípica e genotípica envolvida na patofisiologia da $\mathrm{CMH}$.

Quanto à avaliação fenotípica, observaram-se diferenças significativas entre os valores de Em/Am apenas no corte apical quatro câmaras, em região de base do septo interventricular (4CSB). Neste corte, os valores de Em/Am foram menores nos gatos acometidos pela $\mathrm{CMH}$ do que nos suspeitos e naqueles fenotipicamente normais. Segundo Chetboul et al. (2006b), o Doppler tecidual colorido é capaz de identificar disfunção ventricular longitudinal, nos gatos com $\mathrm{CMH}$, por diminuição na velocidade diastólica precoce
(Em), aumento na velocidade diastólica tardia (Am) e presença de contrações pós-sistólicas tanto no ápice quanto na base do coração. No presente trabalho, apesar de não se observarem diferenças significativas nos valores de Em e de Am isoladamente, pôde-se notar redução significativa na relação Em/Am nos indivíduos acometidos, o que, de certa forma, representa a diminuição das velocidades diastólicas precoces (Em) e aumento nas diastólicas tardias (Am) nesses indivíduos.

Foi observada correlação positiva entre a frequência cardíaca e as velocidades miocárdicas Sm e Em em diversos segmentos avaliados. Quanto maior a frequência cardíaca, maiores as velocidades sistólicas (Sm) e diastólicas (Em) avaliadas pelo Doppler tecidual colorido. Porém, observou-se que as ondas Em foram mais influenciadas pela frequência cardíaca que as ondas Sm neste método ecocardiográfico. No estudo de McDonald et al. (2007), à semelhança dos resultados obtidos no presente trabalho, concluiu-se que quando ocorre fusão das ondas Em e Am devido à taquicardia, os valores de Em desses animais são normalmente superiores aos dos indivíduos sem a fusão de ondas.

Segundo Nagueh et al. (2001), velocidade Em do anel mitral inferior a $13 \mathrm{~cm} / \mathrm{s}$ teve sensibilidade de $100 \%$ e especificidade de $90 \%$ para a identificação de humanos portadores da mutação para a $\mathrm{CMH}$, mas sem hipertrofia ventricular. Ho et al. (2002), em estudo similar, obtiveram ponto de corte ótimo de $13,5 \mathrm{~cm} / \mathrm{s}$, porém com precisão diagnóstica boa apenas nos jovens. Porém, diferentemente destes trabalhos citados, no estudo atual não foi possível detectar um valor de velocidade miocárdica abaixo do qual a identificação da CMH e/ou da mutação fosse alta. Portanto, estes resultados novamente indicam que mais estudos são necessários para estabelecer a utilidade do Doppler tecidual na detecção de anormalidades, antes do desenvolvimento de hipertrofia ventricular (Graziano \& Acquatella 2007).

Na interpretação do teste de variabilidade intraobservador, não houve evidências de que as diferenças encontradas entre as medidas do Doppler tecidual colorido, realizadas em tempos diferentes e pelo mesmo observador, fossem distintas entre si. Portanto, a variabilidade intraobservador foi pequena, comprovando a boa repetibilidade do método. Segundo Sampedrano et al. (2006), a modalidade do Doppler tecidual colorido possui repetibilidade e reprodutibilidade satisfatórias em gatos saudáveis.

As velocidades miocárdicas sistólicas e diastólicas avaliadas tanto pelo Doppler tecidual pulsado quanto pelo Doppler tecidual colorido foram maiores nas regiões de base em relação às regiões médias do miocárdio, e maiores no endocárdio em relação ao epicárdio. Segundo Pinto (2007), num coração normal, a região endocárdica apresenta uma velocidade de contração maior em relação à região epicárdica; e a velocidade é maior na base em relação à porção média e ao ápice na avaliação pelo corte longitudinal, de acordo com o que foi o observado na maioria dos segmentos miocárdicos avaliados no presente estudo.

A ocorrência de fusão das ondas diastólicas Em e Am, em qualquer corte ecocardiográfico avaliado tanto pelo Doppler tecidual pulsado quanto pelo Doppler tecidual co- 
lorido, esteve significativamente correlacionada com a frequência cardíaca. Quanto maior a frequência cardíaca do animal avaliado, maior a ocorrência de fusão das ondas Em e Am. Segundo McDonald (2005) e Boon (2011), nos gatos, muitas vezes, o fluxo mitral da ecocardiografia convencional apresenta uma fusão das ondas E e A, em decorrência da alta frequência cardíaca, dificultando sua avaliação. De acordo com o trabalho atual, esta influência da frequência cardíaca também existe na ecocardiografia tecidual e dificulta a interpretação e análise das ondas diastólicas avaliadas pelo Doppler tecidual pulsado.

Ao avaliar os valores da relação E/Em obtidos no presente estudo (em região basal parietal e basal septal no corte apical quatro câmaras), observou-se relação E/Em superior a 15, indicativa de pior prognóstico, em quatro indivíduos (método do Doppler tecidual pulsado) e em 14 indivíduos (método do Doppler tecidual colorido). Durante a realização do presente estudo, um paciente apresentou morte súbita (fêmea homozigota suspeita para a $\mathrm{CMH}$ à avaliação inicial) e outro, complicações decorrentes da cardiomiopatia (fêmea homozigota fenotipicamente normal à avaliação inicial). Estes dois animais apresentavam valores de E/Em superiores a 15 na maioria dos cortes ecocardiográficos, avaliados pelo Doppler tecidual pulsado e Doppler tecidual colorido, descritos anteriormente. Segundo McMahon et al. (2004), relação E/Em elevada é indicativa de maior risco de morte súbita, parada cardíaca e taquicardia ventricular em crianças com CMH. De acordo com Graziano \& Acquatella (2007), a relação E/Em associa-se, também, à capacidade funcional e ao consumo de oxigênio em adultos com CMH. Vários trabalhos demonstram uma relação direta entre o quociente E/Em e a pressão telediastólica do ventrículo esquerdo (Efthimiadis et al. 2007). Segundo o trabalho de Ommen et al. (2000), relação E/Em superior a 15 (ou seja, onda $E$ alta e onda Em de baixa velocidade) corresponde a uma pressão diastólica de ventrículo esquerdo elevada.

As principais limitações deste estudo referem-se ao pequeno número de animais analisados. 0 número de homozigotos foi pequeno, impedindo comparação estatística e reduzindo a chance de detecção de alterações precoces nesses indivíduos. Outra limitação diz respeito à faixa etária jovem da população. Apesar da maioria dos animais se encontrar na faixa de maior ocorrência da $\mathrm{CMH}$, alguns animais apresentavam idade inferior a 1,5 anos $(10,52 \%$ da população de estudo). Portanto, não se pode excluir a possibilidade da existência de disfunções diastólicas mais evidentes ou até mesmo de CMH se estes animais fossem avaliados em idades mais avançadas, embora o objetivo do presente trabalho seja a avaliação de alterações precoces.

Em muitos gatos, a taquicardia prejudicou avaliações e interpretações ecocardiográficas. A fusão de ondas E e A foi frequente nos diferentes cortes avaliados, prejudicando as análises.

Evidencia-se, portanto, que o Doppler tecidual é uma ferramenta muito importante em futuros estudos envolvendo prevenção e terapia das cardiomiopatias, inclusive em seus estágios mais precoces. Até o presente momento, diante destes resultados apresentados, o Doppler tecidual ainda não pode ser classificado como uma ferramenta muito importante no diagnóstico precoce das cardiomiopatias, mas as alterações encontradas nos gatos acometidos e suspeitos poderiam sim auxiliar na conduta clínica. Apesar da grande expectativa em relação ao uso do Doppler tecidual para a identificação precoce de indivíduos portadores da $\mathrm{CMH}$, ainda há necessidade de estudos mais extensos e com maior número de indivíduos.

Agradecimentos.- À Fundação de Amparo à Pesquisa do Estado de São Paulo - FAPESP pelo Auxílio Pesquisa concedido; aos criadores de gatos da raça Maine Coon e respectivos gatis do estado de São Paulo que participaram da pesquisa; ao Centro de Diagnóstico e Especialidades Provet, pela realização da dosagem do T4 total; ao laboratório Veterinary Cardiac Genetics Laboratory da Washington State University, pela realização dos testes genéticos.

\section{REFERÊNCIAS}

Abecasis J., Dourado R., Arroja I., Azevedo J. \& Silva A. 2009. Utility of tissue characterization in apical hypertrophic cardiomyopathy diagnosis. Eur. J. Echocardiography 10(2):325-328.

Abbott J.A. \& McLean H.N. 2006. Two-dimensional echocardiographic assessment of the feline left atrium. J. Vet. Intern. Med. 20:111-119.

Baty C.J. 2004. Feline hypertrophic cardiomyopathy: an update. Vet. Clin. Small Anim. 34:1227-1234.

Boon J.A. 2011. Manual of Veterinary Echocardiography. Wiley-Blackwell, West Sussex. 610p.

Brown S., Atkins C., Bagley R., Carr A., Cowgill L., Davidson M., Egner B., Elliott J., Henik R., Labato M., Littman M., Polzin D., Ross L., Snyder P. \& Stepien R. 2007. Guidelines for the identification, evaluation and management of systemic hypertension in dogs and cats. J. Vet. Intern. Med. 21(3):542-558.

Buchanan J. W. \& Bücheler J. 1995. Vertebral scale system to measure canine heart size in radiographs. J. Am. Vet. Med. Assoc. 206:194-199.

Chetboul V., Blot S., Sampedrano C.C., Thibaud J.L., Granger N., Tissier R., Bruneval P., Gaschen F., Gouni V., Nicolle A.P. \& Pouchelon J.L. 2006a. Tissue Doppler imaging for detection of radial and longitudinal myocardial dysfunction in a family of cats affected by dystrophin-deficient hypertrophic muscular dystrophy. J. Vet. Intern. Med. 20:640-647.

Chetboul V., Sampedrano C.C., Gouni V., Nicolle A.P. \& Pouchelon J.L. 2006b. Two-dimensional color tissue Doppler imaging detects myocardial dysfunction before occurrence of hypertrophy in a young Maine Coon cat. Vet. Radiol. Ultrasound 47(3):295-300.

Connolly D.J., Cannata J., Boswood A., Archer J., Groves E.A. \& Neiger R. 2003. Cardiac troponin I in cats with hypertrophic cardiomyopathy. J. Feline Med. Surg. 5:209-216.

Efthimiadis G.K., Giannakoulas G., Parcharidou D.G., Karvounis H.I., Mochlas S.T., Styliadis I.H., Papadopoulos C.E., Kounatiadis P., Pliakos C.I., Parcharidis G.E. \& Louridas G.E. 2007. Clinical significance of tissue Doppler imaging in patients with hypertrophic cardiomyopathy. Circulation J. 71:897-903.

Ferasin L. 2009a. Feline myocardial disease. J. Feline Med. Surg. 11(1): 3-13.

Ferasin L. 2009b. Feline myocardial disease- diagnosis, prognosis and clinical management. J. Feline Med. Surg. 11:183-194.

Fox P.R. 1999. Feline cardiomyopathies, p.621-641. In: Fox P.R., Sisson D. \& Moise N.S. (Eds), Textbook of Canine and Feline Cardiology: principles and clinical practice. W.B. Saunders, Philadelphia.

Fries R., Heaney A.M. \& Meurs K.M. 2008. Prevalence of the myosin-binding protein $\mathrm{C}$ mutation in Maine Coon cats. J. Vet. Intern. Med. 22:893896.

Fuentes V.L. 2006. Cardiomyopathy: establishing a diagnosis, p.301-310. In: August J.F. (Eds), Consultation in Feline Internal Medicine. $5^{\text {th }}$ ed. Elsevier Saunders, St Louis. 
Gandjbakhch E., Gackowski A., Montcel S.T., Isnard R., Hamroun A., Richard P., Komajda M. \& Charron P. 2010. Early identification of mutation carriers in familial hypertrophic cardiomyopathy by combined echocardiography and tissue Doppler imaging. Eur. Heart J. 31:1599-1607.

Graziano P. \& Acquatella H. 2007. Ecocardiografia clínica na cardiomiopatia hipertrófica: importância do diagnóstico diferencial na tomada de decisões, p.681-712. In: Silva C.R.S. (Ed.), Ecocardiografia: princípios e aplicações clínicas. Revinter, Rio de Janeiro.

Gundler S., Tidholm A. \& Häggström J. 2008. Prevalence of myocardial hypertrophy in a population of asymptomatic Swedish Maine coon cats. Acta Vet. Scand. 50(22):1-6.

Häggström J. 2003. Hypertrophic cardiomyopathy in cats: it used to be simple. J. Feline Med. Surg. 5:139-141.

Ho C.Y., Sweitzer N.K., McDonough B., Maron B.J., Casey S.A., Seidman J.G., Seidman C.E. \& Solomon S.D. 2002. Assessment of diastolic function with Doppler tissue imaging to predict genotype in preclinical hypertrophic cardiomyopathy. Circulation 105:2992-2997.

Kato T.S., Izawa H., Komamura K., Noda A., Asano H., Nagata K., Hashimoto S., Oda N., Kamiya C., Kanzaki H., Hashimura K., Ueda H.I., Murohara T., Kitakaze M. \& Yokota M. 2007. Heterogeneity of regional systolic function detected by tissue Doppler imaging is linked to impaired global left ventricular relaxation in hypertrophic cardiomyopathy. Heart 94:1302-1306.

Kittleson M.D. 2009. The genetics of HCM-mutant cats. Canadian ACVIM Forum Veterinary Proceedings, p.120-121.

Kittleson M.D., Meurs K.M., Munro M.J., Kittleson J.A., Liu S.K., Pion P.D. \& Towbin J.A. 1999. Familial hypertrophic cardiomyopathy in Maine Coon cats: an animal model of human disease. Circulation 99:3172-3180.

Koffas H., McEwan J.D., Corcoran B.M., Moran C.M., French A., Sborost V., Simpson K. \& McDicken W.N. 2006. Pulsed tissue Doppler imaging in normal cats and cats with hypertrophic cardiomyopathy. J. Vet. Intern. Med. 20:65-77.

Koffas H., McEwan J.D., Corcoran B.M., Moran C.M., French A., Sborost V., Simpson K., Anderson T. \& McDicken W.N. 2008. Colour M-mode tissue Doppler imaging in healthy cats and cats with hypertrophic cardiomyopathy. J. Small Anim. Pract. 49:330-338.

McDonald K. 2005. Myocardial disease: feline, p.1328-1341. In: Ettinger S.T. \& Feldman E.C. (Eds), Textbook of Veterinary Internal Medicine. Elsevier Saunders, St Louis.

McDonald K.A., Kittleson M.D., Larson R.F., Kass P.H., Klose T. \& Wisner E.R. 2006a. The effect of ramipril on left ventricular mass, myocardial fibrosis, diastolic function, and plasma neurohormones in Maine Coon cats with familial hypertrophic cardiomyopathy without heart failure. J. Vet. Intern. Med. 20:1093-1105.

McDonald K.A., Kittleson M.D., Nolen T.G., Larson R.F. \& Wisner E.R. 2006b. Tissue Doppler imaging and gradient echo cardiac magnetic resonance imaging in normal cats and cats with hypertrophic cardiomyopathy. J. Vet. Intern. Med. 20:627-634.

McDonald K.A., Kittleson M.D., Kass P.H. \& Meurs K.M. 2007. Tissue Doppler imaging in Maine Coon cats with a mutation of myosin binding protein C with or without hypertrophy. J. Vet. Intern. Med. 21:232-237.

McDonald K.A., Kittleson M.D. \& Kass P.H. 2008. Effect of spironolactone on diastolic function and left ventricular mass in Maine Coon cats with familial hypertrophic cardiomyopathy. J. Vet. Intern. Med. 22:335-341.

McMahon C.J., Nagueh S.F., Pignatelli R.H., Denfield S.W., Dreyer W.J., Price J.F., Clunie S., Bezold L.I., Hays A.L., Towbin J.A. \& Eidem B.W. 2004. Characterization of left ventricular diastolic function by tissue Doppler imaging and clinical status in children with hypertrophic cardiomyopathy. Circulation 109(14):1756-1762.

McTaggart D.R. 2002. Tissue Doppler imaging in hypertrophic cardiomyopathy without left ventricular hypertrophy. Heart, Lung and Circulation 11:92-94.

Meurs K.M., Sanchez X., David R.M., Bowles N.E., Towbin J.A., Reiser P.J., Kittleson J.A., Munro M.J., Dryburgh K., McDonald K.A. \& Kittleson M.D. 2005. A cardiac myosin binding protein C mutation in the Maine Coon cat with familial hypertrophic cardiomyopathy. Human Mol. Genetics 14(23):3587-3593.
Meurs K.M., Norgard M.M., Ederer M.M., Hendrik K.P. \& Kittleson M.D. 2007. A substitution mutation in the myosin binding protein $C$ gene in Ragdoll hypertrophic cardiomyopathy. Genomics 90:261-264.

Meurs K.M., Norgard M.M., Kuan M., Häggström J., Kittleson M. 2009. Analysis of 8 sarcomeric candidate genes for feline hypertrophic cardiomyopathy mutations in cats with hypertrophic cardiomyopathy. J. Vet. Intern. Med. 23:840-843.

Nagueh S.F., Bachinski L.L., Meyer D., Hill R., Zoghbi W.A., Tam J.W., Quinones M.A., Roberts R. \& Marian A.J. 2001. Tissue Doppler imaging consistently detects myocardial abnormalities in patients with hypertrophic cardiomyopathy and provides a novel means for early diagnosis before and independently of hypertrophy. Circulation 104:128130.

Neter J., Kutner M.H., Nachtsheim C.J. \& Wasserman W. 1996. Applied linear statistical models. $4^{\text {th }}$ ed. Times Mirror Higher Education Group, USA. $1408 \mathrm{p}$.

Oki T., Mishiro Y., Yamada H., Onose Y., Matsuoka M., Wakatsuki T., Tabata T. \& Ito S. 2000. Detection of left ventricular regional relaxation abnormalities and asynchrony in patients with hypertrophic cardiomyopathy with hypertrophic cardiomyopathy with the use of tissue Doppler imaging. Am. Heart J. 139:487-502.

Ommen S.R., Nishimura R.A., Appleton M.D., Miller M.D., Oh J.K., Redfield M.M. \& Tajik M.D. 2000. Clinical utility of Doppler echocardiography and tissue Doppler imaging in the estimation of left ventricular filling pressures: a comparative simultaneous Doppler-catheterization study. Circulation 102:1788-1794.

Pinto F.J. 2007. Doppler tecidual, p.147-154. In: Silva C.R.S. (Ed.), Ecocardiografia: princípios e aplicações clínicas. Revinter, Rio de Janeiro.

Sampedrano C.C., Chetboul V., Gouni V., Nicolle A.P., Pouchelon J.L. \& Tissier R. 2006. Systolic and diastolic myocardial dysfunction in cats with hypertrophic cardiomyopathy or systemic hypertension. J. Vet. Intern. Med. 20:1106-1115.

Sampedrano C.C., Chetboul V., Mary J., Tissier R., Abitbol M., Serres F., Gouni V., Thomas A. \& Pouchelon J.L. 2009. Prospective echocardiography and tissue Doppler imaging screening of a population of Maine Coon cats tested for the A31P mutation in the myosin-binding protein C gene: a specific analysis of the heterozygous status. J. Vet. Intern. Med. 23:91-99.

Severino S., Caso P., Cicala M., Galderisi M., Simone L., D’Andrea D., D’Errico A. \& Mininni N. 2000. Involvement of right ventricle in left ventricular hypertrophic cardiomyopathy: analysis by pulsed Doppler tissue imaging. Eur. J. Echocardiogr. 1:281-288.

Siegel S. \& Castellan N.J. 1988. Nonparametric statistics. $2^{\text {nd }}$ ed. McGraw-Hill, New York. 399p.

Simpson K.E., Devine B.C., Gunn-Moore D.A., French A.T., Dukes-McEwan J., Koffas H., Moran C.M. \& Corcoran B.M. 2007. Assessment of the repeatability of feline echocardiography using conventional echocardiography and spectral pulse-wave Doppler tissue imaging techniques. Vet. Radiol. Ultrasound 48(1):58-68.

Simpson K.E., Gunn-Moore D.A., Shaw D.J., French A.T., Dukes-McEwan J., Moran C.M. \& Corcoran B.M. 2008. Pulsed-wave Doppler tissue imaging velocities in normal geriatric cats and geriatric cats with primary or systemic diseases linked to specific cardiomyopathies in humans, and the influence of age and heart rate upon these velocities. J. Feline Med. Surgery 8:1-12.

Thomas W.P., Gaber C.E., Jacobs G.J., Kaplan P.M., Lombard C.W., Moise N.S. \& Moses B.L. 1992. Recommendations for standards in transthoracic two-dimensional echocardiography in the dog and cat. J. Vet. Intern. Med. 7(4):247-252.

Tilley L.P. 1992. Essentials of canine and feline electrocardiography. $3^{\text {rd }}$ ed. Lea and Febiger, Philadelphia. 470p.

Toro R., Perez-Isla L., Doxastaquis G., Barba M.A., Gallego A.R., Pintos G., Barbados F.J., Mangas A. \& Zamorano J.L. 2009. Clinical usefulness of tissue Doppler imaging in predicting preclinical Fabry cardiomyopathy. Int. J. Cardiol. 132:38-44.

Ware W. 2007. Myocardial disease of the cat, p.300-319. In: Ware W. (Ed.), 
Cardiovascular Disease in Small Animal Medicine. Manson Publishing, London.

Wess G., Sarkar R. \& Hartmann K. 2010. Assessment of left ventricular systolic function by strain imaging echocardiography in various stages of feline hypertrophic cardiomyopathy. J. Vet. Intern. Med. 24(6):1375-1382.

Wess G., Schinner C., Weber K., Küchenhoff H. \& Hartmann K. 2010. Association of $\mathrm{A} 31 \mathrm{P}$ and $\mathrm{A} 74 \mathrm{~T}$ polymorphisms in the myosin binding pro- tein C3 gene and hypertrophic cardiomyopathy in Maine coon and other breed cats. J. Vet. Intern. Med. 24:527-532.

Wynne J. \& Braunwald E. 2003. Cardiomiopatias e miocardites, p.18101869. In: Braunwald E., Zipes D.P. \& Libby P.(Eds), Tratado de Medicina Cardiovascular. Roca, São Paulo.

Yu C.M.,Sanderson J.E., Marwick T.H. \& Oh J.K. 2007. Tissue Doppler imaging. J. Am. College Cardiol. 49(19):1903-1914. 